\title{
Invasive Paget's Disease of the Breast: A case Report
}

\author{
Sang Eun Park, M.D. ${ }^{1}$, Yong Hwa Eom, M.D., Ph.D. ${ }^{1}$, Se Jeong Oh, M.D., Ph.D. ${ }^{2}$ \\ Department of Surgery, Division of Breast-Thyroid Surgery, ${ }^{1}$ Incheon St. Mary's Hospital, College of Medicine, The Catholic University of Korea, \\ Incheon; ${ }^{2}$ Seoul St. Mary's Hospital, College of Medicine, The Catholic University of Korea, Seoul, Korea
}

Intramammary Paget's disease is an uncommon disease in which $90 \%$ of the cases are accompanied by invasive or noninvasive ductal carcinoma. It comprises approximately $0.7 \%-4.3 \%$ of all breast cancers. Typically, extramammary Paget's disease is accompanied by dermal invasion; however, in intramammary Paget's disease, dermal invasion through the basement membrane of the skin is very rare. Intramammary Paget's disease with dermal invasion has been reported infrequently worldwide, and its management and prognosis remain unknown. We report a case of intramammary Paget's disease with dermal invasion in a 64-year-old woman, accompanied by review of the literature.

Key Words: Breast, Dermis, Mammary, Paget's disease

\section{INTRODUCTION}

Paget's disease was first reported by James Paget in 1874 [1], with intramammary Paget's disease presenting as a reddish, eczematous patch or ulceration in the nipple-areolar complex. Histologically, tumor cells (Paget's cells) are observed within the epidermis, and it has been reported that $90 \%$ of cases are accompanied by invasive or noninvasive ductal carcinoma [1]. Intramammary Paget's disease is rare and comprises about $0.7 \%-4.3 \%$ of all breast cancers, and accounts for $6.5 \%$ of the total instances of Paget's disease [2]. In general, intramammary Paget's disease is often diagnosed in post-operative tissue specimens, rather than through clinical symptoms. Recently, the incidence of intramammary Paget's disease has decreased [3].

The pathogenesis of intramammary Paget's disease continues to be debated, with two main theories involved. The epidermotropic theory states that Paget's cells are ductal carcinoma cells that have migrated from the underlying breast parenchyma to the nipple epidermis, known as Pagetoid spread [1]. In the in situ transformation theory, the Paget's cells arise as malignant cells in the nipple epidermis, independent from any other pathologic process within the breast parenchyma [1].

Pathologically, a Paget's cell is a large, pale-staining cell with round

Correspondence: Yong Hwa Eom, M.D., Ph.D.

Division of Breast-Thyroid Surgery, Department of Surgery, Incheon St. Mary's Hospital, College of Medicine, The Catholic University of Korea, 56 Dongsoo-ro, Bupyeong-gu, Incheon 21431, Korea

Tel: +82-32-280-5609, Fax: +82-32-280-5988, E-mail: hurrah98@naver.com

Received: Oct 5, 2018 Revised: Aug 29, 2019 Accepted: Nov 24, 2019 or oval nuclei and large nucleoli, located between the normal keratinocytes on the nipple epidermis. Paget's cells do not invade through the dermal basement membrane and therefore are a form of carcinoma in situ [4]

Intramammary Paget's disease with dermal invasion has been reported rarely worldwide, and its management and prognosis remain unknown. We report this rare case with a review of the literature.

\section{CASE REPORT}

A 64-year-old female patient presented with painless, desquamative, erythematous patches in the right nipple-areolar complex (Figure 1). The lesion had grown for approximately 2-3 years. The patient had been on medication under the diagnosis of hypertension and dyslipidemia for 7-8 years, and she had no remarkable family history.

A physical examination revealed an infiltrating lesion with erosion of $6.0 \times 5.0 \mathrm{~cm}$ in size located on the right nipple-areolar complex. There were no palpable masses in the breast, and no palpable axillary or supraclavicular lymph nodes. She underwent skin punch biopsy and was diagnosed with Paget's disease.

All laboratory data were unremarkable. Mammography showed skin thickening and nipple inversion in the right nipple-areolar complex (Figure 2). Magnetic resonance imaging (MRI) showed a circumferential skin enhancement about $6.5 \times 6.0 \mathrm{~cm}$ in size, and an underlying mass of the breast parenchyma was not observed (Figure 3). There were no specific findings on the abdominal computed tomography 


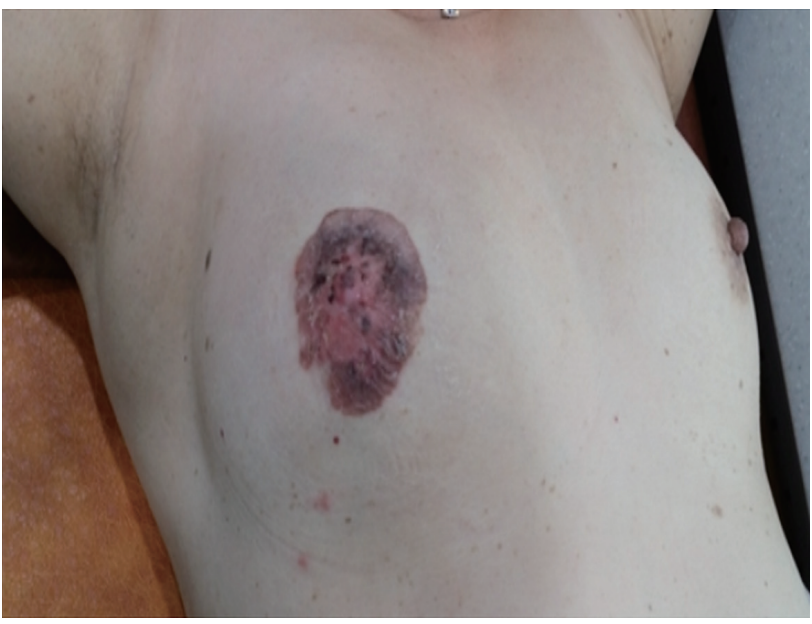

Figure 1. Physical findings. Desquamative and erythematous patches with erosion located on the right nipple-areolar complex.

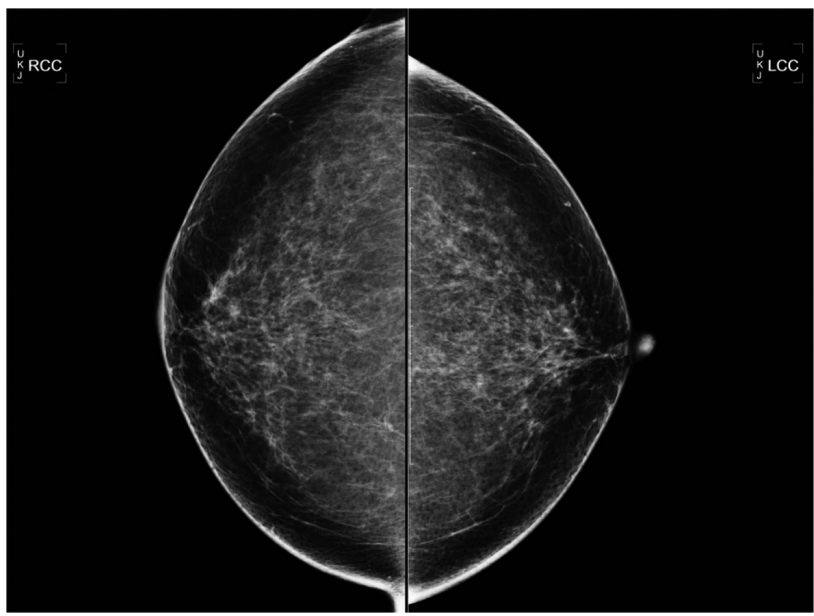

Figure 2. Mammography findings. The craniocaudal view mammogram showed a skin thickening and nipple inversion in the right nipple-areolar complex.

scan, chest x-ray, and bone scan

The patient underwent a right total mastectomy with ipsilateral sentinel lymph node biopsy, and there was no lymph node metastasis on the frozen section. Macroscopically, the resected specimen revealed a discolored, eczematoid skin lesion on the entire right nipple-areolar complex. No clear tumor mass was found in the breast (Figure 4). Microscopically, a poorly demarcated, irregular tumor of $6.0 \times 5.5 \times 0.2 \mathrm{~cm}$ in size was revealed. Margins were sufficiently negative. A histological analysis revealed large, pale-staining cells with round or oval nuclei and large nucleoli, called Paget's cells. Invasion of the cells into the dermis was observed (Figure 5). There was no lym-

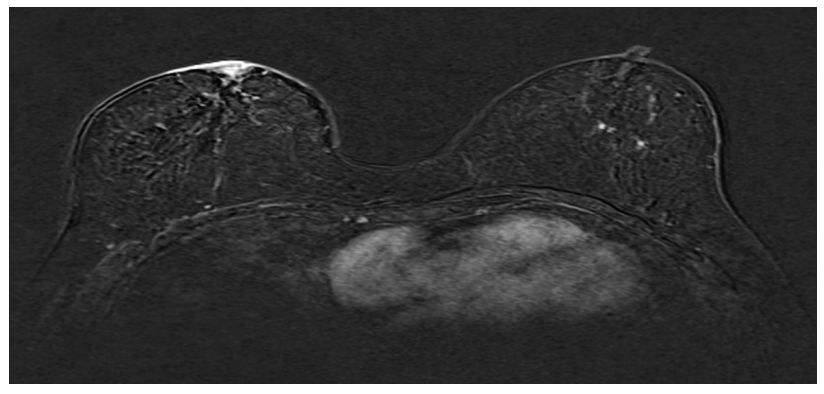

Figure 3. Magnetic resonance imaging (MRI) findings. The MRI showed a circumferential skin enhancement approximately $6.5 \times 6.0 \mathrm{~cm}$ in size. An underlying mass of breast parenchyma was not observed.

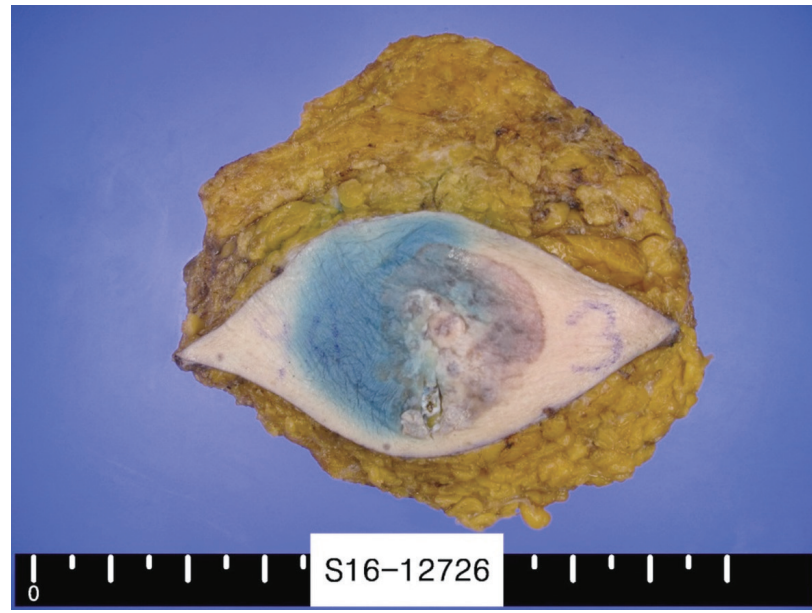

Figure 4. Gross appearance of the mastectomy specimen. A discolored and eczematoid skin lesion approximately $6.0 \times 5.0 \mathrm{~cm}$ in size was observed on the entire right nipple-areolar complex.

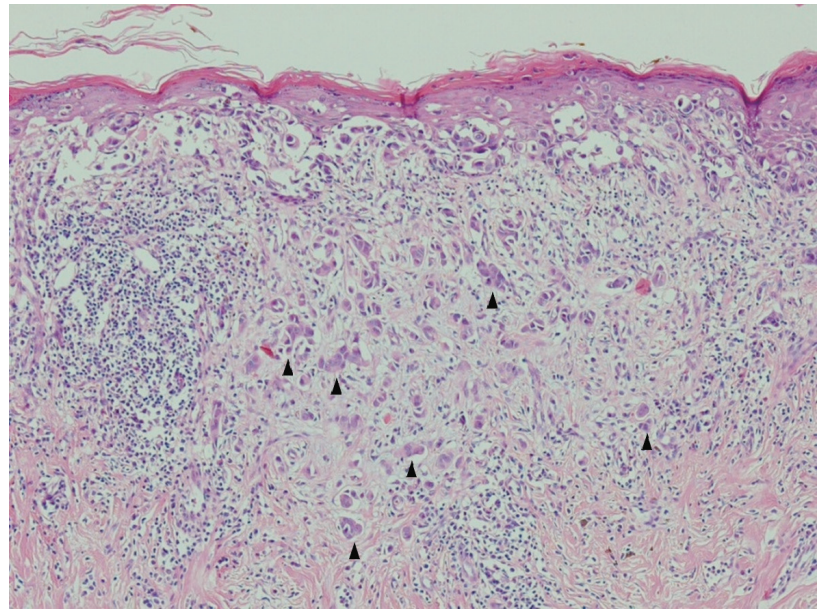

Figure 5. Microscopic findings of the specimen. The epidermis is infiltrated by large pleomorphic cells, called Paget's cells. Paget's cells have abundant cytoplasm and large pleomorphic nuclei, and occasionally the cells have an eccentric nucleus and atypical mitosis. Invasion of the Paget's cells into the dermis was observed (arrows, H\&E stain, $\times 200$ ). 

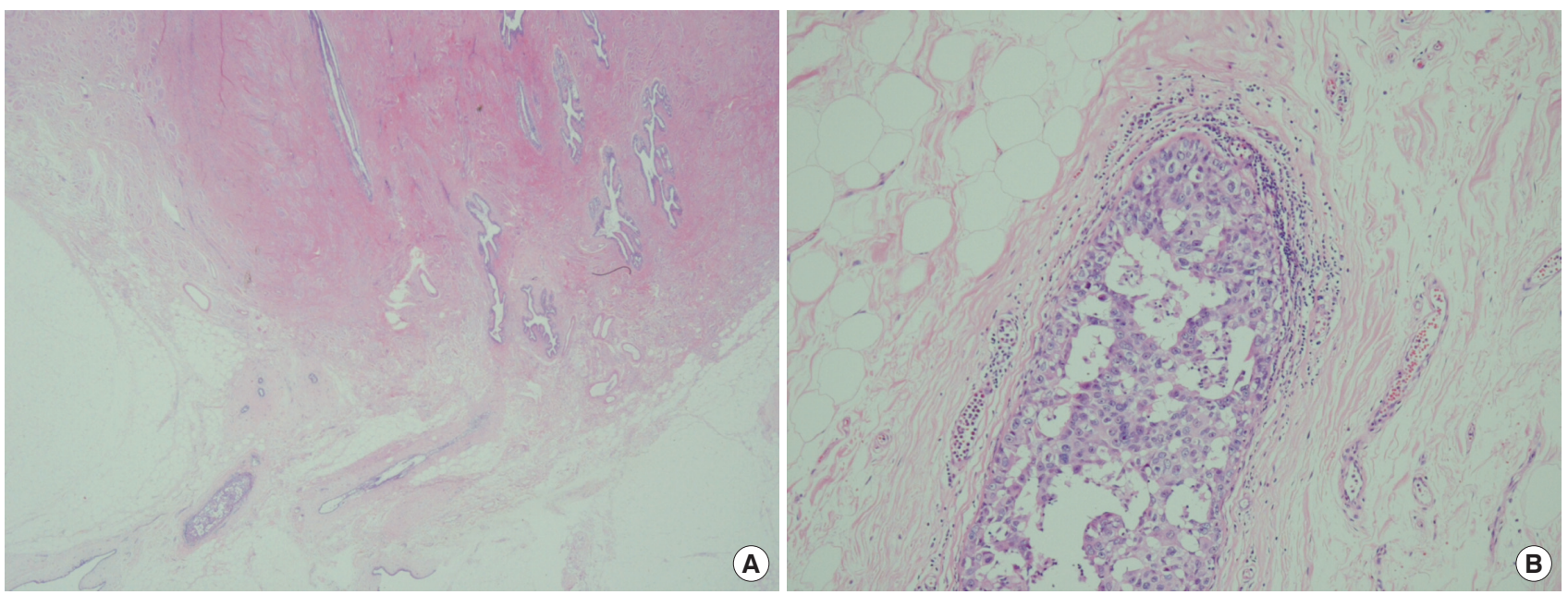

Figure 6. Microscopic findings of the specimen. Low power microscopic findings of the specimen (A, H\&E stain, $\times 40)$, and incidentally detected ductal carcinoma in situ was observed at another central portion ( $B, H \& E$ stain, $\times 100)$.

phovascular invasion, but lactiferous duct involvement was observed. An incidentally detected ductal carcinoma in situ was observed at another central portion. (cribriform type, $0.2 \times 0.1 \times 0.1 \mathrm{~cm}, \mathrm{ER}[+], \mathrm{PR}[-]$, HER2[3+])(Figure 6A, 6B).

After surgery, the patient underwent 4 cycles of Adriamycin plus cyclophosphamide chemotherapy and is currently taking hormone therapy. During the 24 months follow-up period, the patient is on hormone therapy without local recurrence or distant metastasis.

This case study was approved by our Institutional Review Board (IRB No. OC20ZISI0039).

\section{DISCUSSION}

Intramammary Paget's disease is an uncommon disease in which $90 \%$ of cases are accompanied by invasive or noninvasive ductal carcinoma. It comprises approximately $0.7 \%-4.3 \%$ of all breast cancers. According to a study by Ozerdem et al. [5], ductal carcinoma in situ is the most common underlying tumor in the breast with intramammary Paget's disease, comprising approximately $67 \%$ of cases. Our patient also demonstrated ductal carcinoma in situ components in the post-operative specimen.

The morphological and clinical features of intra- and extramammary Paget's disease are similar; however, they differ in invasiveness. In extramammary Paget's disease, superficial invasion into the dermis has been reported in up to $16 \%$ of patients, and dermal invasion predicts regional lymph node metastasis and a poor prognosis. Hatta et al. $[6,7]$ suggests that a depth of dermal invasion $>1 \mathrm{~mm}$ in the case of extramammary Paget's disease is associated with an increased risk of lymph node metastasis. Intramammary Paget's disease generally shows intra-epidermal manifestation, although in very rare cases, tumor cells within the epidermis invade through the basement membrane of the skin into the dermis.

When biopsy of the nipple-areolar complex is positive for Paget's disease, breast MRI is recommended to define the extent of disease and identify additional diseases. When there is intramammary Paget's disease only, it is managed as a ductal carcinoma in situ.

Traditionally, management of intramammary Paget's disease has been considered to be total mastectomy with ipsilateral axillary dissection [8]. In recent years; however, breast conserving surgery is recommended [1]. Systemic therapy is recommended according to the pathological stage and biological characteristics of the underlying cancer. There is no clear information on the clinical outcome or post-operative management of invasive intramammary Paget's disease due to its rare incidence.

There have been a few reports of systemic chemotherapy in invasive intramammary Paget's disease. According to a case report by Sueta et al. [9], a 66-year-old female patient was diagnosed with intramammary Paget's disease and underwent total mastectomy. In the post- operative specimen, dermal invasion of Paget's cells was detected. Eighteen months after the operation, she was diagnosed with local recurrence 
of intramammary Paget's disease with ipsilateral axillary lymph node and lung metastasis. She was treated with twelve courses of weekly paclitaxel in combination with trastuzumab. A complete response was obtained 11 months after initiation of chemotherapy.

In another report by Saluja et al. [10], the authors performed systemic chemotherapy in a patient experiencing invasive intramammary Paget's disease with axillary lymph node metastasis. As in our case, an underlying ductal carcinoma in situ was observed in the post- operative specimen. The patient received an adjuvant chemotherapy of paclitaxel and cyclophosphamide and experienced no recurrence after 20 months of follow-up.

We also performed systemic chemotherapy with adriamycin and cyclophosphamide for prevention of recurrence and distance metastasis. During the 24 months follow-up period, we did not detect any local recurrence or distant metastasis. However, we do not have clear evidence regarding the treatments effectiveness, due to the rare incidence of the disease and a lack of research.

There are a few reports of invasive intramammary Paget's disease in domestic and overseas cases. There are also several studies comparing invasive intramammary Paget's disease with the noninvasive type. According to a study by Duan et al. [11], retrospective slide reviews from 1985 to 2010 found invasive intramammary Paget's disease in 6 cases out of 146 patients. Five patients were associated with invasive ductal carcinoma and ductal carcinoma in situ, and one was not associated with cancer. Management was based on the underlying carcinoma, and no clear prognostic significance was found. The value of sentinel lymph node biopsy was also questionable.

In Korea, according to a study by Lee et al. [12], invasive intramammary Paget's disease was found in 16 cases from a total of 205 cases reviewed retrospectively for 20 years, from 1994 to 2013. Of these, 12 cases were accompanied by invasive carcinoma and 3 cases were accompanied by ductal carcinoma in situ. The horizontal extent was clearly wider in invasive intramammary Paget's disease, but there was no significant difference in distant metastasis or disease-related death. In comparison, prognosis of invasive intramammary Paget's disease was similar to that of noninvasive intramammary Paget's disease.

We have presented a rare case of invasive intramammary Paget's disease. Due to its scarcity, there is still little information regarding its management and prognosis. In this case, our patient underwent sentinel lymph node biopsy and additional systemic chemotherapy; how- ever, there is no clear evidence for the effectiveness of this treatment in the surrounding literature. As of now, local recurrence or distant metastasis has not occurred.

Further studies are necessary to determine the usefulness of sentinel lymph node biopsy and establish management protocols for invasive intramammary Paget's disease. Long-term follow-up will also be needed in diagnosed patients.

\section{CONFLICT OF INTEREST}

The authors declare that they have no competing interests.

\section{REFERENCES}

1. Kang DH, Gye CS, Kang BJ, Ko SS, Kang SH, Ko ES, et al. The breast. 3rd ed. Seoul: Ilchokak; 2013. p.1055-9.

2. Kanitakis J. Mammary and extramammary Paget's disease. J Eur Acad Dermatol Venereol 2007;21:581-90.

3. Chen CY, Sun LM, Anderson BO. Paget disease of the breast: changing patterns of incidence, clinical presentation, and treatment in the U.S. Cancer 2006;107:1448-58.

4. Oncohema Key: Paget's Disease. Nora M. Hansen. https://oncohemakey.com/pagets-disease. Accessed Aug 18th, 2018.

5. Ozerdem U, Swistel A, Antonio LB, Hoda SA. Invasive Paget disease of the nipple: a brief review of the literature and report of the first case with axillary nodal metastases. Int J Surg Pathol 2014;22:566-9.

6. Hatta N, Morita R, Yamada M, Echigo T, Hirano T, Takehara K, et al. Sentinel lymph node biopsy in patients with extramammary Paget's disease. Dermatol Surg 2004;30:1329-34.

7. Hatta N, Yamada M, Hirano T, Fujimoto A, Morita R. Extramammary Paget's disease: treatment, prognostic factors and outcome in 76 patients. Br J Dermatol 2008;158:313-8.

8. Paone JF, Baker RR. Pathogenesis and treatment of Paget's disease of the breast. Cancer 1981;48:825-9.

9. Sueta A, Yamamoto Y, Arase K, Iizaka M, Kuriwaki K, Inoue K. A case of mammary Paget's disease with early systemic recurrence after operation. Gan To Kagaku Ryoho 2010;37:1329-32.

10. Saluja K, Sahoo S. Invasive Paget disease of the nipple of luminal-B subtype with axillary lymph node metastasis in a 60-year-old white 
woman. Lab Med 2015;46:332-7.

11. Duan X, Sneige N, Gullett AE, Prieto VG, Resetkova E, Andino LM, et al. Invasive Paget disease of the breast: clinicopathologic study of an underrecognized entity in the breast. Am J Surg Pathol 2012;36:
1353-8.

12. Lee HW, Kim TE, Cho SY, Kim SW, Kil WH, Lee JE, et al. Invasive Paget disease of the breast: 20 years of experience at a single institution. Hum Pathol 2014;45:2480-7. 